TITLE:

\title{
Fast multiple inversion for stress analysis from fault-slip data
}

$\operatorname{AUTHOR}(S)$ :

Sato, Katsushi

CITATION:

Sato, Katsushi. Fast multiple inversion for stress analysis from fault-slip data. Computers \& Geosciences 2012, 40: 132-137

ISSUE DATE:

2012-03

URL:

http://hdl.handle.net/2433/153282

RIGHT:

(c) 2011 Elsevier Ltd.; この論文は出版社版でありません。引用の際には 出版社版をご確認ご利用ください。; This is not the published version. Please cite only the published version. 


\title{
Fast multiple inversion for stress analysis from fault-slip data
}

\author{
Katsushi Sato ${ }^{\mathrm{a}, *}$ \\ ${ }^{a}$ Division of Earth and Planetary Sciences, Kyoto University, Kyoto 606-8502, Japan
}

\begin{abstract}
The multiple inverse method is widely used to invert multiple stress tensors from fault-slip data caused by polyphase tectonics. A practical problem of the method is the time-consuming computation related to its iterative procedure. This paper describes a way of accelerating the computation by replacing an exhaustive grid search for the optimal stress tensor by direct calculation using an analytical solution. Furthermore, a technique to reduce noise in the result was developed based on the estimation of instabilities of solutions.
\end{abstract}

Keywords: stress tensor inversion, tectonic stress, algorithm, even-determined problem, deviatoric stress space

\section{Introduction}

Stress tensor inversion methods are widely used to infer tectonic stress 3 state from fault-slip data. Input fault data are collected from geological out4 crops, seismic focal mechanisms, rock core samples and underground images 5 obtained by three-dimensional seismic surveys. Among the variety of meth-

\footnotetext{
${ }^{*}$ Corresponding author.

Email address: k_sato@kueps.kyoto-u.ac.jp (Katsushi Sato)
} 
6 ods the multiple inverse method (Yamaji, 2000), hereafter abbreviated as 7 MIM, has an advantage in separating multiple stress tensors from a mix8 ture of geological faults yielded from spatial or temporal change of tectonic 9 stress state. This method has been used by many researchers in various 10 regions (e.g., Yamada and Yamaji, 2002; Yamaji, 2003; Sippel et al., 2009; 11 Chan et al., 2010) and further methodological improvement is now ongoing. MIM has been extended to analyse seismic focal mechanisms without a priori specification of fault planes from paired orthogonal nodal planes (Otsubo 14 et al., 2008), improved to objectively recognise multiple solutions by means of clustering techniques (Otsubo and Yamaji, 2006) and enhanced in its resolution through development of uniform computational grid (Sato and Yamaji, 2006b; Yamaji and Sato, 2011).

A fault-slip data set is described as heterogeneous when it includes faults caused by different stresses. A conventional method of stress inversion (e.g., Angelier, 1979) determines an optimal stress tensor for a whole data set, though the solution is meaningless if the data set is heterogeneous. MIM can detect multiple stress tensors through an iterative sampling procedure. When a data set has $N$ faults, MIM extracts a subset including $k$ faults from it and determines an optimal stress tensor for the subset by exhaustive grid search. This process is repeated ${ }_{N} \mathrm{C}_{k}$ times for all possible combinations of $k$-element subsets. A great number of stress tensors are obtained and their concentrations are interpreted as desired tectonic stresses (Fig. 1). This iterative calculation also has an effect of enhancing solutions from natural noisy fault-slip data.

A problem of MIM lies in its computational cost. It takes between a few 
hours and several days to analyse several hundred to a thousand faults by a personal computer. The cost is proportional to the number of fault subsets ${ }_{N} \mathrm{C}_{k}$, which is order of $O\left(N^{k}\right)$ by Landau's symbol. The number of faults in a subset $k$ is empirically set to four or five (Yamaji, 2000). Therefore the cost is $O\left(N^{4}\right)$ or $O\left(N^{5}\right)$. This fact generally limits the total number of faults $N$ up to a thousand.

Each determination of optimal stress for fault subsets is done by exhaustive grid search on 60,000 uniformly spaced stress tensors (Sato and Yamaji, 2006b) by default. This study proposes a direct algorithm for determination of optimal stress tensor. Although the new technique is applicable only to four-element subsets, it calculates the numerous stress solutions several times faster than conventional MIM. A method of noise reduction by estimating instabilities of solutions is also provided.

\section{Method}

\subsection{Wallace-Bott hypothesis}

MIM as well as recent stress tensor inversion techniques is based on an assumption that a fault slips in the direction of shear stress, which is called Wallace-Bott hypothesis (Wallace, 1951; Bott, 1959, illustrated in Fig. 2a). Input data of stress inversion analysis are called fault-slip data which contain fault plane orientations, slip orientations and shear senses, while the unknown parameters are described by stress tensors. The direction of shear stress on a fault plane depends on four of the six independent components of stress tensor. Let $\boldsymbol{\sigma}$, whose components are denoted by $\sigma_{i j}(i=1$ to $3, j=1$ to 3$)$, be a reduced stress tensor with four degrees of freedom. Two normalisation 


$$
\boldsymbol{v} \cdot \boldsymbol{t}>0
$$

68 where the unit vector $\boldsymbol{b}=\boldsymbol{n} \times \boldsymbol{v}$ is perpendicular to both $\boldsymbol{n}$ and $\boldsymbol{v}$. Eq. (3)

conditions imposed on $\boldsymbol{\sigma}$ can be freely chosen. The first and second invariants are normalised in this study, i.e.

$$
J_{1}=\sigma_{1}+\sigma_{2}+\sigma_{3}=0
$$

$$
J_{2}=-\sigma_{1} \sigma_{2}-\sigma_{2} \sigma_{3}-\sigma_{3} \sigma_{1}=1,
$$

where $\sigma_{1}, \sigma_{2}$ and $\sigma_{3}$ are the principal stress magnitudes $\left(\sigma_{1} \geq \sigma_{2} \geq \sigma_{3}\right.$, compression is positive). Let $\boldsymbol{n}=\left(n_{1}, n_{2}, n_{3}\right)^{\mathrm{T}}$ and $\boldsymbol{v}=\left(v_{1}, v_{2}, v_{3}\right)^{\mathrm{T}}$ be the unit vectors in the directions of fault normal and slip direction, respectively. The superscript $\mathrm{T}$ denotes the transpose of a vector or a matrix. Hereafter all vectors are column vectors. Cauchy's formula gives the traction vector exerted on a fault plane by a stress as $\boldsymbol{t}=\boldsymbol{\sigma} \boldsymbol{n}$. The shear stress is derived by projecting $\boldsymbol{t}$ onto fault plane as $\boldsymbol{\tau}=\boldsymbol{t}-\boldsymbol{n} \boldsymbol{n}^{\mathrm{T}} \boldsymbol{t}$. The Wallace-Bott hypothesis requires $\boldsymbol{\tau}$ to be in the same direction as $\boldsymbol{v}$.

Fry (1999) decomposed the Wallace-Bott condition into

$$
\boldsymbol{b} \cdot \boldsymbol{t}=0
$$

requires the shear stress vector $\boldsymbol{\tau}$ to be parallel to observed slip direction $\boldsymbol{v}$, while Eq. (4) represents the correspondence of shear sense (Fig. 2a). Sato and Yamaji (2006a) introduced the deviatoric stress space to stress inversion analysis, in which reduced stress tensors and fault-slip data are represented 
73 by five-dimensional unit vectors (Fig. 2b). They reformulated Eqs. (3) and 74 (4) as

$$
\vec{\epsilon}^{\prime} \cdot \vec{\sigma}=0
$$

75 and

$$
\vec{\epsilon} \cdot \vec{\sigma}>0
$$

76 respectively. The vectors in Eqs. (5) and (6) are defined as

$$
\vec{\sigma}=\left(\begin{array}{c}
\sigma_{11} / \sqrt{2} \\
\sigma_{22} / \sqrt{2} \\
\sigma_{33} / \sqrt{2} \\
\sigma_{23} \\
\sigma_{31} \\
\sigma_{12}
\end{array}\right), \vec{\epsilon}^{\prime}=\left(\begin{array}{c}
\sqrt{2} b_{1} n_{1} \\
\sqrt{2} b_{2} n_{2} \\
\sqrt{2} b_{2} n_{2} \\
b_{2} n_{3}+b_{3} n_{2} \\
b_{3} n_{1}+b_{1} n_{3} \\
b_{1} n_{2}+b_{2} n_{1}
\end{array}\right), \vec{\epsilon}=\left(\begin{array}{c}
\sqrt{2} v_{1} n_{1} \\
\sqrt{2} v_{2} n_{2} \\
\sqrt{2} v_{2} n_{2} \\
v_{2} n_{3}+v_{3} n_{2} \\
v_{3} n_{1}+v_{1} n_{3} \\
v_{1} n_{2}+v_{2} n_{1}
\end{array}\right) .
$$

77 The normalisation conditions of the stress tensor (Eqs. 1 and 2) and the 78 orthogonality of unit vectors representing fault parameters (Fig. 2a) imply

$$
\sigma_{11}+\sigma_{22}+\sigma_{33}=\epsilon_{1}^{\prime}+\epsilon_{2}^{\prime}+\epsilon_{3}^{\prime}=\epsilon_{1}+\epsilon_{2}+\epsilon_{3}=0
$$

79

$$
|\vec{\sigma}|=\left|\vec{\epsilon}^{\prime}\right|=|\vec{\epsilon}|=1
$$

80 and

$$
\vec{\epsilon}^{\prime} \cdot \vec{\epsilon}=0
$$

81 Eq. (8) means the components of vectors in the direction of $(1,1,1,0,0,0)^{\mathrm{T}}$ 82 are equal to 0 , which allows us to reduce the dimension on six-dimensional 83 vectors to five. According to Eq. (9) the end points of vectors are on the ${ }_{84}$ five-dimensional unit sphere (Fig. 2b). 
The Wallace-Bott condition is geometrically expressed in the deviatoric stress space (Sato and Yamaji, 2006a). A fault-slip datum specifies paired orthogonal vectors $\vec{\epsilon}^{\prime}$ and $\vec{\epsilon}$ (Eq. 10). The unknown stress tensor is constrained so that $\vec{\sigma}$ is perpendicular to $\vec{\epsilon}^{\prime}$ and is in the same hemisphere as $\vec{\epsilon}$ (Eqs. 5 and 6 ). In other words, stress tensors which satisfy the WallaceBott condition correspond to $\vec{\sigma}$ on a half great circle specified by $\vec{\epsilon}^{\prime}$ and $\vec{\epsilon}$ (Fig. 2b), which is called the Fry arc in what follows.

\subsection{Analytical solution}

When we have a number of faults activated by a single stress, their Fry arcs should intersect at a point on the five-dimensional unit sphere. The point corresponds to the optimal stress tensor satisfying Wallace-Bott conditions for all faults. Since natural data contain errors to some extent, intersections of Fry arcs do not generally coincide. MIM searches for optimal points for fault subsets which have small distances to Fry arcs. The candidates of solutions are the uniformly spaced 60,000 grid points (Sato and Yamaji, 2006b). The exhaustive search on the grid causes the computational cost.

The necessary and sufficient number of fault data to determine a stress solution is four, which is equal to the number of unknown stress parameters. This fact corresponds to the geometry in the deviatoric stress space. In order to satisfy the parallel conditions between shear stress vectors and slip directions (Eq. 5) for four faults, a direction perpendicular to four $\vec{\epsilon}^{\prime}$ vectors in the five-dimensional space is uniquely specified by calculating a cross product of them (Fig. 3). Fortunately, the number of faults in a subset of MIM analysis can be set to four. Then the time-consuming grid search can be replaced by a direct calculation of cross product. The replacement 
is expected to save computational time, although the shear sense conditions

(Eq. 6) must be checked separately.

\subsection{Procedure}

The present method of fast multiple inversion, hereafter FMI, takes the following steps.

1. Convert $N$ fault-slip data into $\vec{\epsilon}$ and $\vec{\epsilon}^{\prime}$ vectors.

2. Extract a four-element subset from the whole data.

3. Calculate the five-dimensional cross product of four $\vec{\epsilon}^{\prime}$ vectors to obtain a candidate $\vec{\sigma}$ for the optimal solution.

4. Check the shear sense conditions (Eq. 6) by calculating dot products of $\vec{\sigma}$ and $\vec{\epsilon}$ vectors. If all signs of four dot products are positive or negative, $\vec{\sigma}$ or $-\vec{\sigma}$ is the optimal solution for the subset, respectively. Otherwise, reject the candidate $\vec{\sigma}$ and proceed to 6 .

5. Find the nearest grid point to the optimal solution from 60,000 uniform grid points and cast a vote for the corresponding stress tensor.

6. Repeat procedures 2 to $5{ }_{N} \mathrm{C}_{4}$ times for all possible combinations of fault subsets.

The software of FMI is available at the author's web site (http://www.kueps.kyotou.ac.jp/ ${ }^{\sim}$ web-bs/k_sato/software.html).

Step 5 above is necessary to deal with numerous stress tensors. When $N=100$, for example, we need to find concentrations of ${ }_{100} \mathrm{C}_{4}=3,921,225$ solutions, though step 4 probably reduces the number to some extent. The population of solutions are converted into votes for grid points. The peaks 
of distribution of votes on the five-dimensional unit sphere can be visualised and recognised by viewer software.

Noisy votes in the result of MIM analysis partly comes from heterogeneous fault subsets, for which the optimal solutions are meaningless and expected to be random stress tensors (Yamaji, 2000). Otsubo and Yamaji (2006) proposed a method to reduce such noise by excluding a candidate solution if the distance between corresponding $\vec{\sigma}$ vector and at least one Fry arc is larger than a threshold value. In the present method of FMI step 4 performs the exclusion during the check of shear sense conditions.

Another type of noise can arise from the instability of cross product calculated in step 3 . If four $\vec{\epsilon}^{\prime}$ vectors are not sufficiently linearly independent, i.e., at least two of them are nearly parallel, the direction of their cross product becomes instable. The degree of linear independence is measured by the length of the cross product, which is the volume of four-dimensional parallelepiped spanned by $\vec{\epsilon}^{\prime}$ vectors. The length ranges from 0 to 1 . For the purpose of reducing noisy votes, FMI has an option to weight votes proportionally to the lengths of cross products in the procedure 5 .

\section{Improvement}

\subsection{Test 1: Reduction of calculation time}

Artificial fault-slip data sets were analysed to compare the calculation times of MIM and FMI. The number of faults in a subset $k$ in MIM was set to four. An example of a data set is shown in Fig. 4a. Fault planes are randomly oriented. A half of the faults in a data set is assumed to be activated by stress $\mathrm{A}$ with $\sigma_{1}$-axis at $000 / 00, \sigma_{3}$-axis at $090 / 00$ and $\Phi=0.3$. 
The other half corresponds to stress B with $\sigma_{1}$-axis at $040 / 00, \sigma_{3}$-axis at $130 / 00$ and $\Phi=0.3$. The parameter $\Phi=\left(\sigma_{2}-\sigma_{3}\right) /\left(\sigma_{1}-\sigma_{3}\right)$ is called stress ratio, which ranges from 0 to 1 . $\Phi=0$ for axial compression $\left(\sigma_{1}>\sigma_{2}=\sigma_{3}\right)$ and $\Phi=1$ for axial tension $\left(\sigma_{1}=\sigma_{2}>\sigma_{3}\right)$.

As the result of MIM and FMI analyses, the artificial stresses A and B were successfully detected (Fig. 4b and c). No large difference was found between results of MIM with grid search and FMI with direct calculation as is expected. The time spent for calculation is shown in Fig. 5a for the cases of $N=50$ to 500 . Although the calculation time rapidly increases with the number of data for both methods, FMI was found to be about ten times faster than MIM.

The calculation time for analysis of seismic focal mechanisms was also examined (Fig. 5b). For a four-element subset, the number of possible choices between orthogonal nodal planes is $2^{4}=16$. All choices are regarded as different subsets of faults in both MIM and FMI, of which calculation inevitably requires much longer time than analysis of geological fault data. Fig. 5b clearly shows that FMI is several times faster than MIM.

\subsection{Test 2: Noise reduction}

As is mentioned in Section 2.3, FMI has an option to reduce noisy solutions by weighting them according to the lengths of five-dimensional cross products. This option can reduce noises caused by nearly parallel $\vec{\epsilon}^{\prime}$ vectors which correspond to nearly parallel fault planes and slip directions. In order to test the effect of noise reduction, an artificial fault data set with 100 faults were analysed (Fig. 6). The faults were assumed to be activated by a single stress tensor with stress ratio $\Phi$ of 0.3 and with $\sigma_{1^{-}}$and $\sigma_{3}$-axes oriented 
$340 / 10$ and 160/80, respectively. The normals of fault planes were concentrated at $000 / 45$ and 180/45 with some perturbation, simulating a conjugate fault system.

As the results of MIM (Fig. 6b), FMI (Fig. 6c) and FMI with noise reduction (Fig. 6d), the assumed stress tensor was successfully detected. The difference between methods appeared in the accuracy and precision of solution. The accuracy can be measured by angular stress distance $\Theta$ (Yamaji and Sato, 2006), which is the reformulation of stress difference proposed by Orife and Lisle (2003), between optimal solutions and the assumed stress tensor. MIM resulted in $\Theta=5.38^{\circ}$, while FMI with noise reduction had a higher accuracy of $\Theta=1.61^{\circ}$. The precision was measured by the dispersion of numerous solutions derived from all fault subsets, which can be estimated by the mean distance $\bar{\Theta}$ to the optimal (averaged) solution. FMI with noise reduction was found to have higher precision of $\bar{\Theta}=15.6^{\circ}$ than that of MIM, $\bar{\Theta}=22.7^{\circ}$. The weighting of solutions by the lengths of cross products was confirmed to be effective in reducing noise.

\section{Discussion}

The new method of multiple stress inversion (FMI) was found to accelerate the calculation by a factor of up to 10 without loss of detectability of stress tensors. Moreover, the noise reduction technique is available in FMI analysis. However, the dependence of calculation amount of FMI on the number of fault data is still $O\left(N^{4}\right)$, the same as MIM, as is demonstrated by the rapidly increasing trends of calculation time in Fig. 5. It will take several days to analyse more than a thousand faults by using personal computers. 
The problem is severe especially for seismic focal mechanisms because of the availability of databases accumulating numerous seismic events and the unknown choice between nodal planes. Further reduction of calculation time could be achieved by relaxing the requirement of analysing all possible combinations of fault subsets. We could undertake random sampling of fault subsets to limit the computation effort, which of course requires a careful assessment of degeneration of results.

\section{Acknowledgement}

The author is grateful to Dr. R.J. Lisle and Dr. T.G. Blenkinsop for their detailed reviews and suggestions which improved the manuscript. This work was partly supported by JSPS KAKENHI 21740364.

\section{References}

Angelier, J., 1979. Determination of the mean principal directions of stresses for a given fault population. Tectonophysics 56 (3-4), T17-T26.

Bott, M.H.P., 1959. The mechanics of oblique slip faulting. Geological Magazine 96 (2), 109-117.

Chan, L.S., Shen, W., Pubellier, M., 2010. Polyphase rifting of greater Pearl River Delta region (South China): Evidence for possible rapid changes in regional stress configuration. Journal of Structural Geology 32 (6), 746 754 .

Fry, N., 1999. Striated faults: visual appreciation of their constraint on possible paleostress tensors. Journal of Structural Geology 21 (1), 7-21. 
Orife, T., Lisle, R.J., 2003. Numerical processing of palaeostress results. Journal of Structural Geology 25 (6), 949-957.

Otsubo, M., Yamaji, A., 2006. Improved resolution of the multiple inverse method by eliminating erroneous solutions. Computers \& Geosciences $32(8), 1221-1227$.

Otsubo, M., Yamaji, A., Kubo, A., 2008. Determination of stresses from heterogeneous focal mechanism data: An adaptation of the multiple inverse method. Tectonophysics 457 (3-4), 150-160.

Sato, K., Yamaji, A., 2006a. Embedding stress difference in parameter space for stress tensor inversion. Journal of Structural Geology 28 (6), 957-971.

Sato, K., Yamaji, A., 2006b. Uniform distribution of points on a hypersphere for improving the resolution of stress tensor inversion. Journal of Structural Geology 28 (6), 972-979.

Sippel, J., Scheck-Wenderoth, M., Reicherter, K., Mazur, S., 2009. Paleostress states at the south-western margin of the Central European Basin System - Application of fault-slip analysis to unravel a polyphase deformation pattern. Tectonophysics 470 (1-2), 129 - 146.

Twiss, R.J., Gefell, M.J., 1990. Curved slickenfibers - a new brittle shear sense indicator with application to a sheared serpentinite. Journal of Structural Geology 12 (4), 471-481.

Wallace, R.E., 1951. Geometry of shearing stress and relation to faulting. Journal of Geology 59 (2), 118-130. 
250 Yamada, Y., Yamaji, A., 2002. Determination of palaeostresses from

251 mesoscale shear fractures in core samples using the multi-inverse method.

252 Journal of Petroleum Geology 25 (2), 203-218.

${ }_{253}$ Yamaji, A., 2000. The multiple inverse method: a new technique to separate $254 \quad$ stresses from heterogeneous fault-slip data. Journal of Structural Geology $255 \quad 22(4), 441-452$.

256 Yamaji, A., 2003. Slab rollback suggested by latest Miocene to Pliocene ${ }_{257}$ forearc stress and migration of volcanic front in southern Kyushu, northern Ryukyu arc. Tectonophysics 364 (1-2), 9-24.

259 Yamaji, A., Sato, K., 2006. Distances for the solutions of stress tensor inversion in relation to misfit angles that accompany the solutions. Geophysical 261 Journal International 167 (2), 933-942. Yamaji, A., Sato, K., 2011. A spherical code and stress tensor inversion. Computers \& Geosciences, in press. 


\section{Figure captions}

Figure 1

Schematic figure illustrating the procedure of multiple inverse method (MIM) to detect multiple stress tensors from a heterogeneous fault-slip data set. The data set is a mixture of black and white $f$ symbols representing faults activated by different stresses A and B, respectively. MIM extracts subsets of four or five faults from whole data and determines optimal solutions for them by means of exhaustive grid search on the deviatoric stress space (Sato and Yamaji, 2006b) which is geometrically the surface of five-dimensional unit sphere. Homogeneous subsets are expected to concentrate their votes to the grid points corresponding to stresses A or B, while the meaningless solutions from heterogeneous subsets should be placed randomly.

Figure 2

Wallace-Bott hypothesis as the principle of stress tensor inversion. The slip direction of a fault is assumed to coincide with the shear stress direction exerted by the tectonic stress in question. (a) In the physical space, observable fault parameters are represented by unit vectors $\boldsymbol{v}, \boldsymbol{b}$ and $\boldsymbol{n}$. A correct stress tensor gives shear stress vector $\boldsymbol{\tau}$, which is the projection of traction vector $\boldsymbol{t}$ onto fault plane, in the direction of slip $\boldsymbol{v}$. (b) Schematic figure of deviatoric stress space. Wallace-Bott hypothesis is geometrically expressed as the constraint on stress tensor represented by $\vec{\sigma}$ from a fault-slip datum. The fault parameters $\vec{\epsilon}$ and $\vec{\epsilon}^{\prime}$ specify a half great circle called the Fry arc (bold line) on which $\vec{\sigma}$ vector is required to lie. 
Figure 3

Schematic figure illustrating how to calculate the direct solution of stress tensor inversion. When we have four fault-slip data, four $\vec{\epsilon}^{\prime}$ vectors are specified in the five-dimensional deviatoric stress space. The parallel conditions between fault-slip directions and shear stress vectors require $\vec{\sigma}$ vector representing stress tensor to be perpendicular to all four $\vec{\epsilon}^{\prime}$ vectors. The analytical solution to this even-determined problem can be uniquely obtained as the direction of five-dimensional cross product of $\vec{\epsilon}^{\prime}$ vectors. Note that four $\vec{\epsilon}^{\prime}$ vectors must be linearly independent in the five-dimensional space, although this schematic figure looks as if they were two-dimensionally coplanar owing to lack of dimension. The white circle spanned by them represents not a two-dimensional circle but a four-dimensional space.

Figure 4

An example of results of the test to examine the computational cost of FMI. (a) Artificial fault-slip data containing 50 faults of which half is activated by stress $\mathrm{A}$ and the other half is activated by stress B. Tangent-lineation diagram (Twiss and Gefell, 1990) in lower-hemisphere and equal-area projection. Arrows plotted at poles of fault planes indicate slip directions of footwall blocks. (b) Result of MIM. Paired stereograms show orientations of $\sigma_{1^{-}}$and $\sigma_{3}$-axes. Colours of symbols indicate stress ratio $\Phi$. In this figure 300 stress tensors out of 60,000 grid points are plotted, which got more votes from fault subsets than the others. The assumed stresses A and B were correctly detected. (c) Result of FMI in similar plot as (b). Note that there is no significant difference between results of MIM and FMI. 
Figure 5

Comparison of calculation times of MIM and FMI. Horizontal axis is the number of faults analysed. (a) Analysis of geological faults. FMI works about ten times faster than MIM, although the calculation times of both methods increase rapidly with the number of data. (b) FMI is also faster in analysis of seismic focal mechanisms, although they require much longer time than geological faults because of unknown choice of nodal planes.

\section{Figure 6}

The result of analysis to test the effect of noise reduction. (a) Artificial 100 fault-slip data assumed to be activated by a single stress with $\Phi=0.3$. Open squares are principal stress axes plotted on lower-hemisphere and equalarea stereogram. Arrows show the slip directions of footwall blocks plotted at poles of fault planes (tangent-lineation diagram). (b) Result of MIM. (c) Result of FMI. (d) Result of FMI with noise reduction. See Fig. 4 for explanation of plots. $\Phi$ values show stress ratios of optimal solutions of which principal orientations are plotted as open squares. The accuracies of the optimal solutions were measured by $\Theta$ values which are distances from the assumed stress. $\bar{\Theta}$ is the dispersion of solutions obtained from fault subsets as a measure of precision. Stress tensors of which votes are more than $1.5 \%$ of their maximum are plotted. Note that higher accuracy and precision was achieved by noise reduction in FMI. 


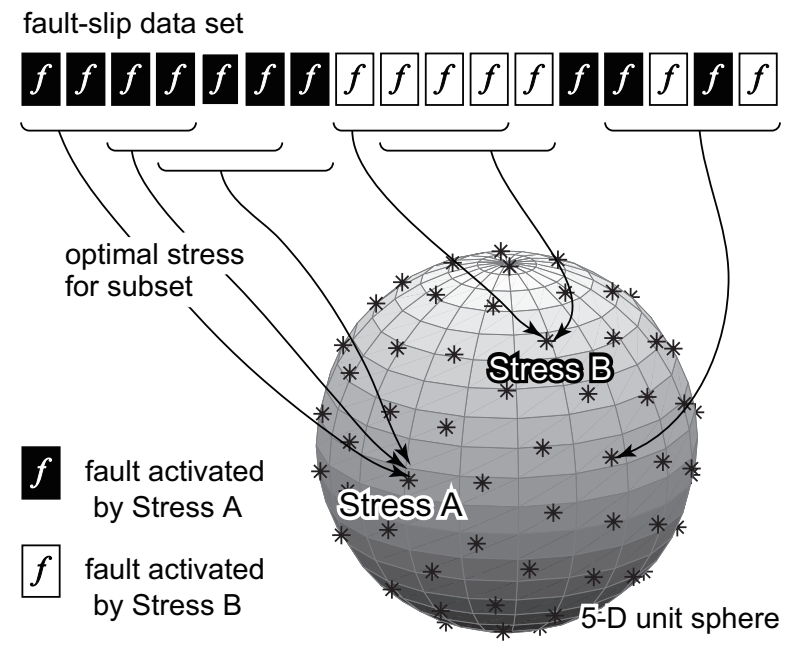

Figure 1: Sato.

(a)

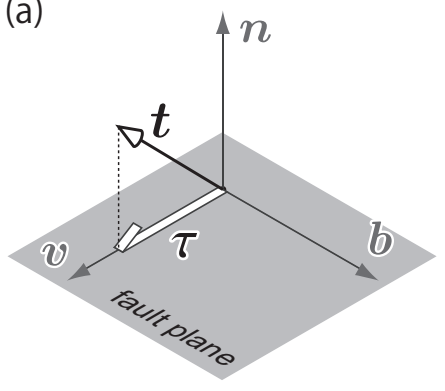

(b)

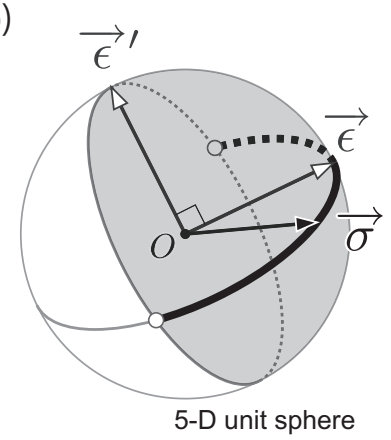

Figure 2: Sato. 


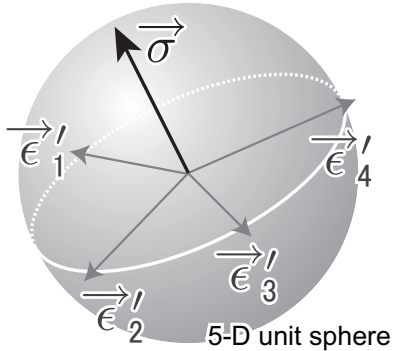

Figure 3: Sato. 

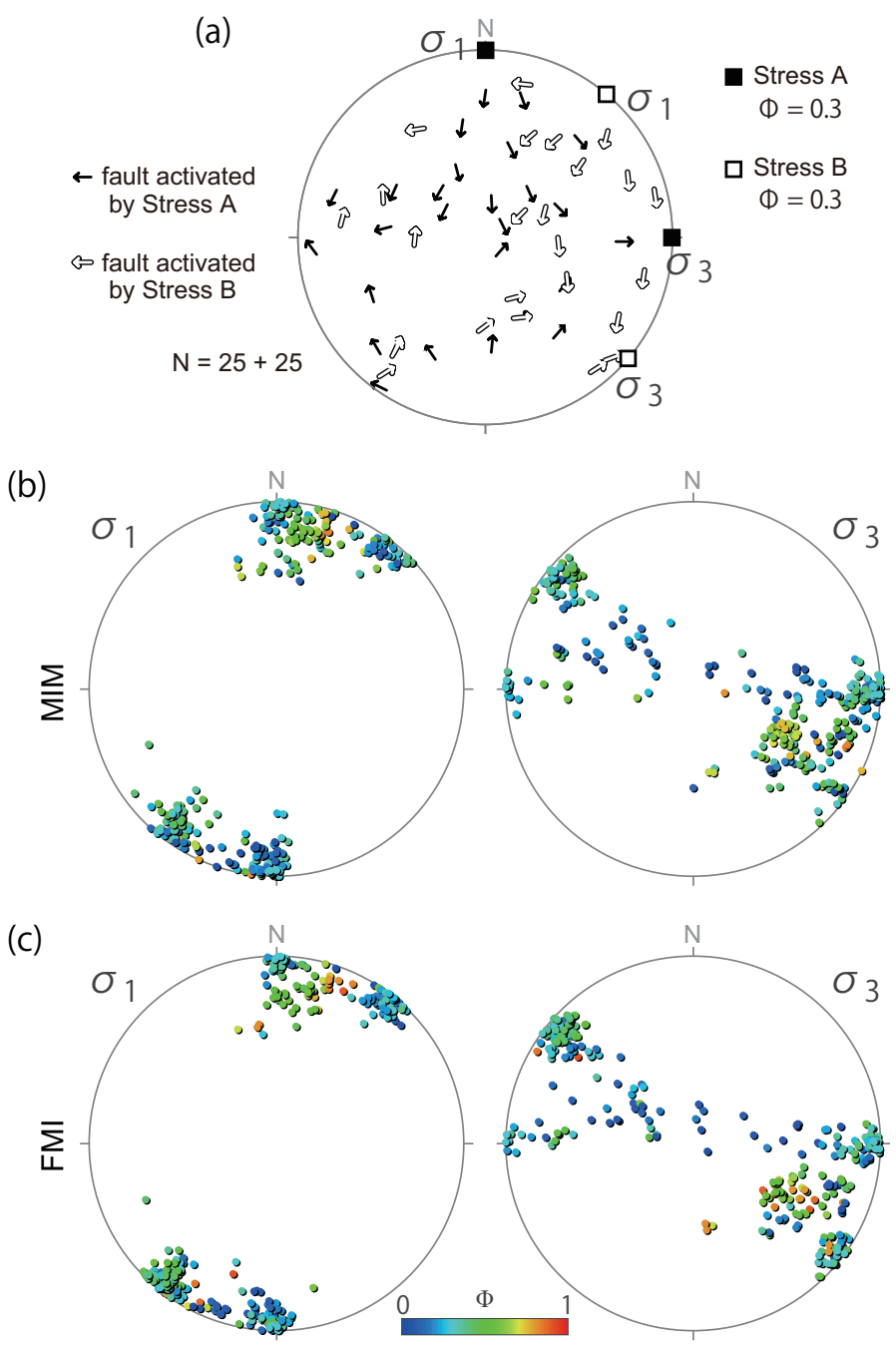

Figure 4: Sato. 
(a) geological fault

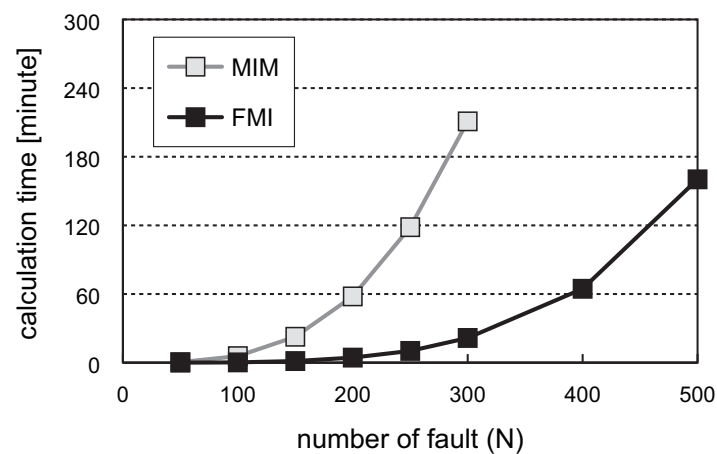

(b) focal mechanism

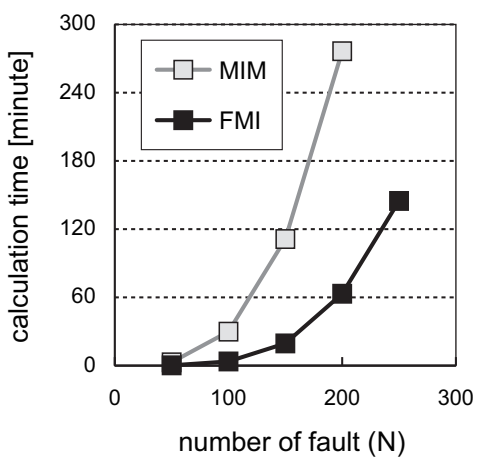

Figure 5: Sato. 


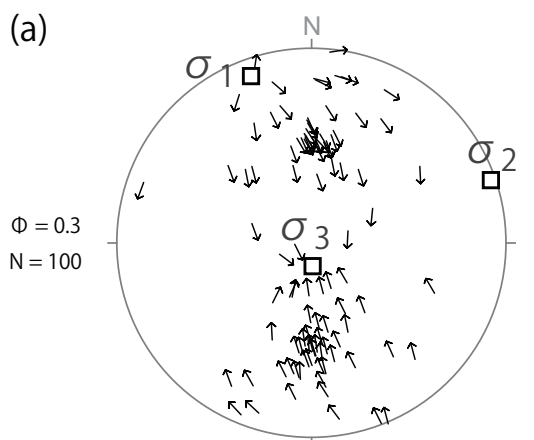

(b)

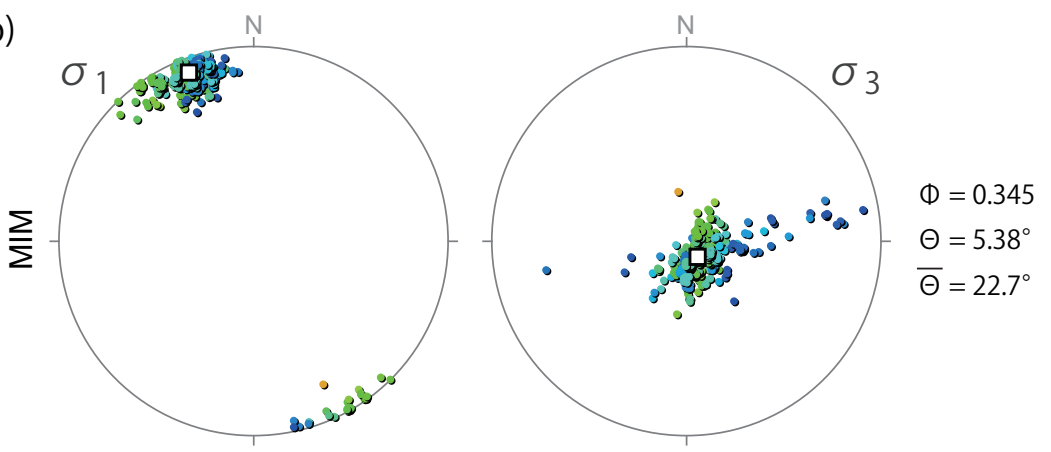

(c)
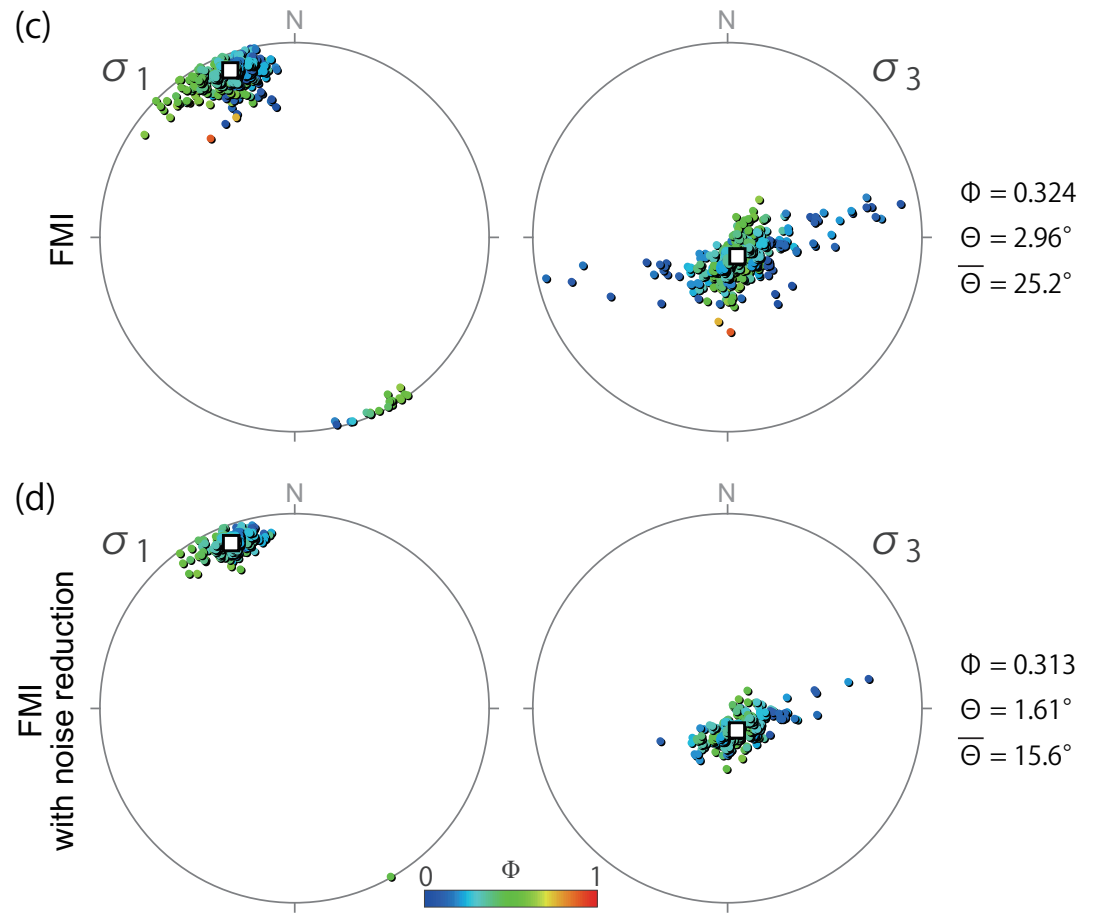

Figure 6: Sato. 